\title{
An Overview of Cinchona Alkaloids in Chemistry
}

\author{
Choong Eui Song
}

\section{1}

\section{Brief History}

Cinchona alkaloids (Figure 1.1), isolated from the bark of several species of cinchona trees, are the organic molecules with the most colorful biography [1]. Their history dates back to the early seventeenth century when they were first introduced into the European market after the discovery of the antimalarial property of cinchona bark and the subsequent isolation of its active compound, quinine, by Pierre-Joseph Pelletier and Joseph Bienaimé Caventou in 1820. Since then, cinchona alkaloids (especially, quinine) have played a pivotal medicinal role in human society for over 300 years. Approximately 700 metric tons of cinchona alkaloids is now extracted from the bark of Cinchona ledgeriana annually. Nearly half of this is used in the food and beverages industry as a bitter additive, and much of the remaining quinine and quinidine is used as an important antimalarial drug and muscle relaxant compound and as a cardiac depressant (antiarrhythmic), respectively.

The role of cinchona alkaloids in organic chemistry was firmly established with the discovery of their potential as resolving agents by Pasteur in 1853, which ushered in an era of racemate resolutions by the crystallization of diastereomeric salts [3]. Today, there are countless examples in which cinchona alkaloids are used as chiral resolving agents [4]. Besides the classical resolution process, significant progress has also been made in the past two decades in the field of cinchona-based enantioseparation, as well as in their use as enantioselective analytical tools (Chapter 13). The considerable effort made to accomplish the stereoselective synthesis of quinine over the past 150 years, which was initially triggered by the supply problem caused by political vagaries of the producing countries, has also undoubtedly laid the foundation for much of modern organic chemistry [5]. However, possibly the most interesting application of cinchona alkaloids in chemistry resides in their ability to promote enantioselective transformations in both homogeneous and heterogeneous catalyses (Chapters 2-11). The first asymmetric reaction carried out using a cinchona base was published by Bredig and Fiske [6] as early as in 1912. These two German chemists reported 

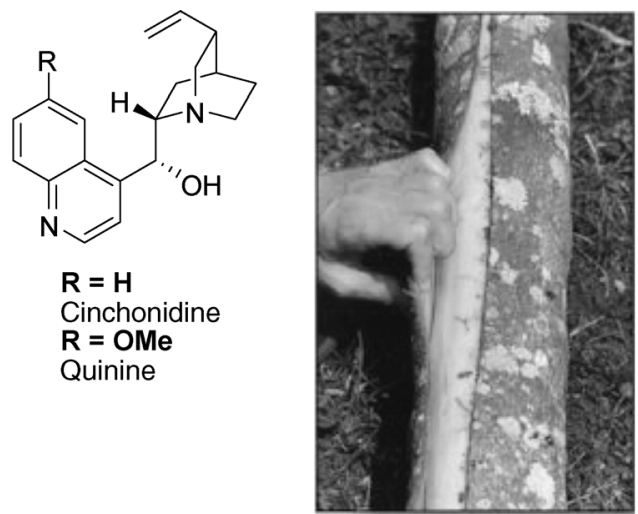

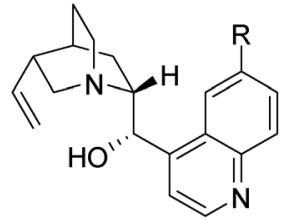

$\mathbf{R}=\mathbf{H}$

Cinchonine

$\mathrm{R}=\mathrm{OMe}$

Quinidine

Figure 1.1 Quinine and other cinchona alkaloids are extracted from the bark of the cinchona tree [2], which is mainly cultivated in Africa, Latin America, and Indonesia. Approximately 700 metric tons of cinchona alkaloids is harvested annually.

that the addition of $\mathrm{HCN}$ to benzaldehyde is accelerated by the pseudoenantiomeric alkaloids, quinine and quinidine, and that the resulting cyanohydrins are optically active and are of opposite chirality. However, the optical yields achieved were in the range of $<10 \%$ ee. After about four decades, Pracejus was first to obtain useful levels of enantioselectivity (74\% ee) by using $O$-acetylquinine as a catalyst ( $1 \mathrm{~mol} \%$ ) in the addition of methanol to phenylmethylketene, affording (-)- $\alpha$-phenyl methylpropionate [7]. Two decades later (in the late 1970s and early 1980s) after Pracejus' seminal study, Wynberg and coworkers began a new era in asymmetric catalysis driven by cinchona alkaloids [8]. Their extensive studies on the use of cinchona alkaloids as chiral Lewis base/nucleophilic catalysts demonstrated that this class of alkaloids could serve as highly versatile catalysts for a broad spectrum of enantioselective transformations (e.g., conjugate additions and the addition of ketenes to carbonyl compounds, resulting in $\beta$-lactones). Since their pioneering studies, the popularity of cinchona derivatives in asymmetric catalysis has increased considerably. During the late 1980s and early 1990s, quite successful examples in terms of the catalytic activity and enantioselectivity have been reported, where the asymmetry was induced by cinchona alkaloids. In particular, Sharpless and coworkers developed the osmium-catalyzed asymmetric dihydroxylation (AD) of olefins [9], which is one of the reactions that has had the greatest impact on synthetic chemistry and for which Sharpless was awarded the Nobel Prize in chemistry in 2001. Furthermore, since 2000, explosively expanding interest in chiral organocatalysis as a new stream of catalysis [10] has sparked a second renaissance in the use of cinchona alkaloids as organocatalysts. Thus, nowadays, cinchona alkaloids and their derivatives are classified as the most "privileged organic chirality inducers," efficiently catalyzing nearly all classes of organic reactions in a highly stereoselective fashion (Chapters 5-11). 


\section{2}

\section{Active Sites in Cinchona Alkaloids and Their Derivatives}

As mentioned in the previous section, nowadays, readily available and inexpensive cinchona alkaloids with pseudoenantiomeric forms, such as quinine and quinidine or cinchonine and cinchonidine, are among the most privileged chirality inducers in the area of asymmetric catalysis. The key feature responsible for their successful utility in catalysis is that they possess diverse chiral skeletons and are easily tunable for diverse types of reactions (Figure 1.2). The presence of the 1,2-aminoalcohol subunit containing the highly basic and bulky quinuclidine, which complements the proximal Lewis acidic hydroxyl function, is primarily responsible for their catalytic activity.

The presence of the quinuclidine base functionality makes them effective ligands for a variety of metal-catalyzed processes (Chapters 2-4). The most representative example is the osmium-catalyzed asymmetric dihydroxylation of olefins [9]. The metal binding properties of the quinuclidine nitrogen also allow to use cinchona alkaloids as metal surface modifiers, for example, in the highly enantioselective heterogeneous asymmetric hydrogenation of $\alpha$-keto esters (Chapter 2). Both

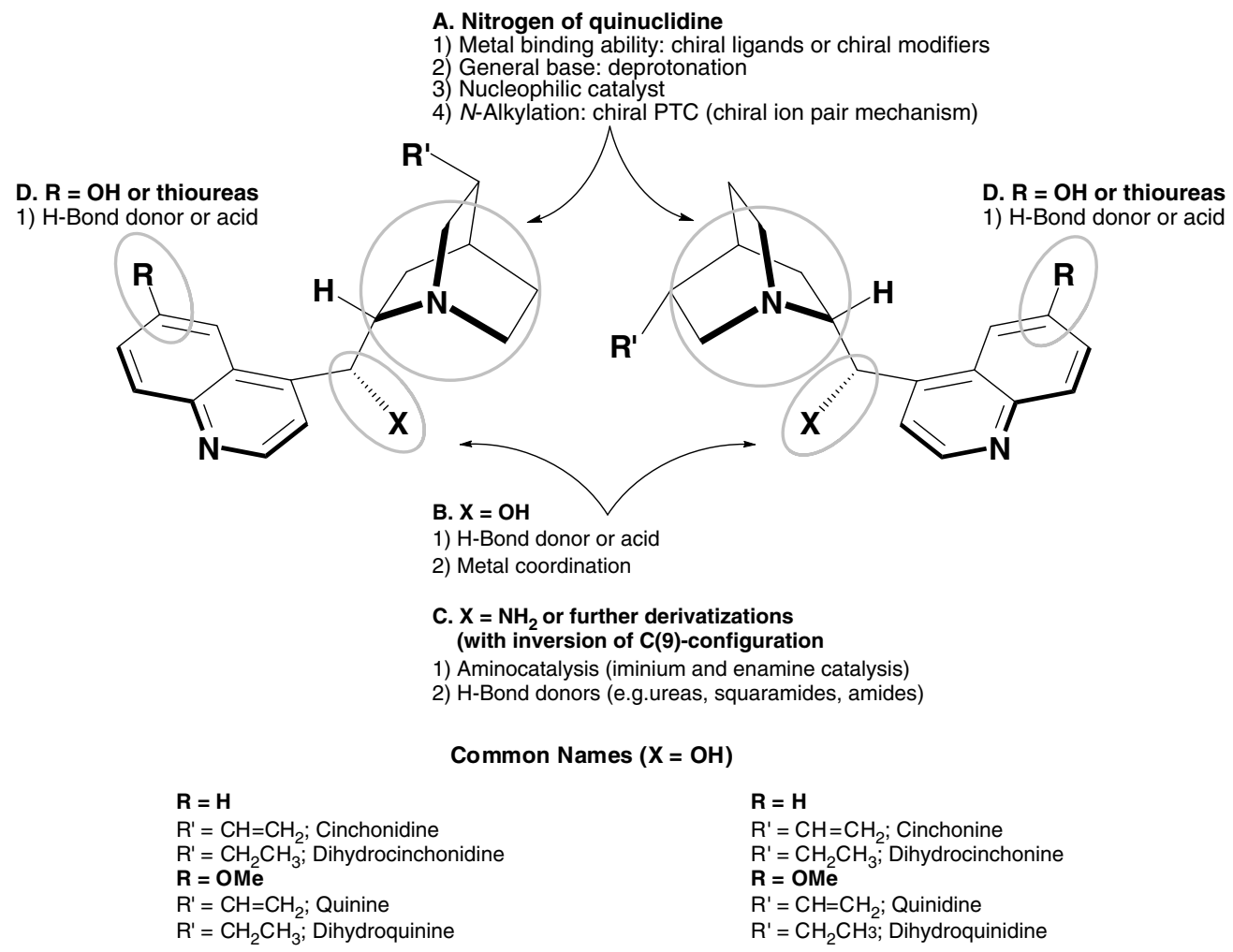

Figure 1.2 Active sites in cinchona alkaloids and their derivatives. 
reactions are classified as ligand-accelerated catalyses (LAC) [11]. In addition to its utility for metal binding, the quinuclidine nitrogen can be used as a chiral base or a chiral nucleophilic catalyst promoting the vast majority of organocatalytic reactions (Chapters 5-11). Finally, the related quaternized ammonium salts of cinchona alkaloids have proved to catalyze numerous reactions under phase-transfer conditions, where asymmetric inductions occur through a chiral ion pairing mechanism between the cationic ammonium species and an anionic nucleophile [12].

The secondary 9-hydroxy group can serve as an acid site or hydrogen bond donor [13]. The derivatization of the $\mathrm{OH}$ group into ureas, amides, and so on, with either the retention or inversion of the configuration, provides a more powerful acidic site or hydrogen bond donor. The $6^{\prime}$-methoxy group of quinine and quinidine can also be readily derivatized to the free $\mathrm{OH}$ group or thiourea moiety, which can serve as an effective H-bond donor. Moreover, the substitution of 9-OH into the free amino group with the inversion of the configuration enables enantioselective aminocatalysis, which includes reactions of the so-called generalized enamine cycle [14] and charge accelerated reactions via the formation of iminium intermediates [15]. Representative examples of modified cinchona alkaloids are depicted in Figure 1.3.

However, in general, these active sites in cinchona alkaloids and their derivatives act in catalysis not independently but cooperatively, that is, they activate the reacting molecules simultaneously. Furthermore, in many cases, the catalysis is also supported by a $\pi-\pi$ interaction with the aromatic quinoline ring or by its steric hindrance.

\section{3}

\section{Structural Information on Cinchona Alkaloids}

Cinchona alkaloids have characteristic structural features for their diverse conformations and self-association phenomena. Therefore, knowledge of their "real structure" in solution can provide original information on the chiral inducing and discriminating ability of these alkaloids.

Conformational investigations of this class of alkaloids, based on computational and spectroscopic methods, have been undertaken with the aim of providing information that would help understand these chiral induction and discrimination processes. Dijkstra et al. were the first to investigate in 1989 the conformational behavior by means of NMR spectroscopic and molecular mechanics (MM) calculations and identify that the $\mathrm{C} 8-\mathrm{C} 9$ and $\mathrm{C} 4{ }^{\prime}-\mathrm{C} 9$ bonds are the most important in determining the overall conformation, resulting in four low-energy conformers (syn-closed, syn-open, anti-closed, and anti-open conformers) (Figure 1.4) [16]. MM calculations showed that the parent alkaloids preferentially adopt an anti-open conformation in nonpolar solvents [16]. More sophisticated ab initio calculations conducted later also revealed that anti-open is the most stable conformer in apolar solvents $[17,18]$. In polar solvents, two other conformers, syn-closed and anti-closed, are strongly stabilized compared to the anti-open conformer, due to the greater support provided by their large dipole moments [18]. For example, in polar solvents, the fraction of cinchonidine adopting a closed conformation is more than $50 \%$ at 

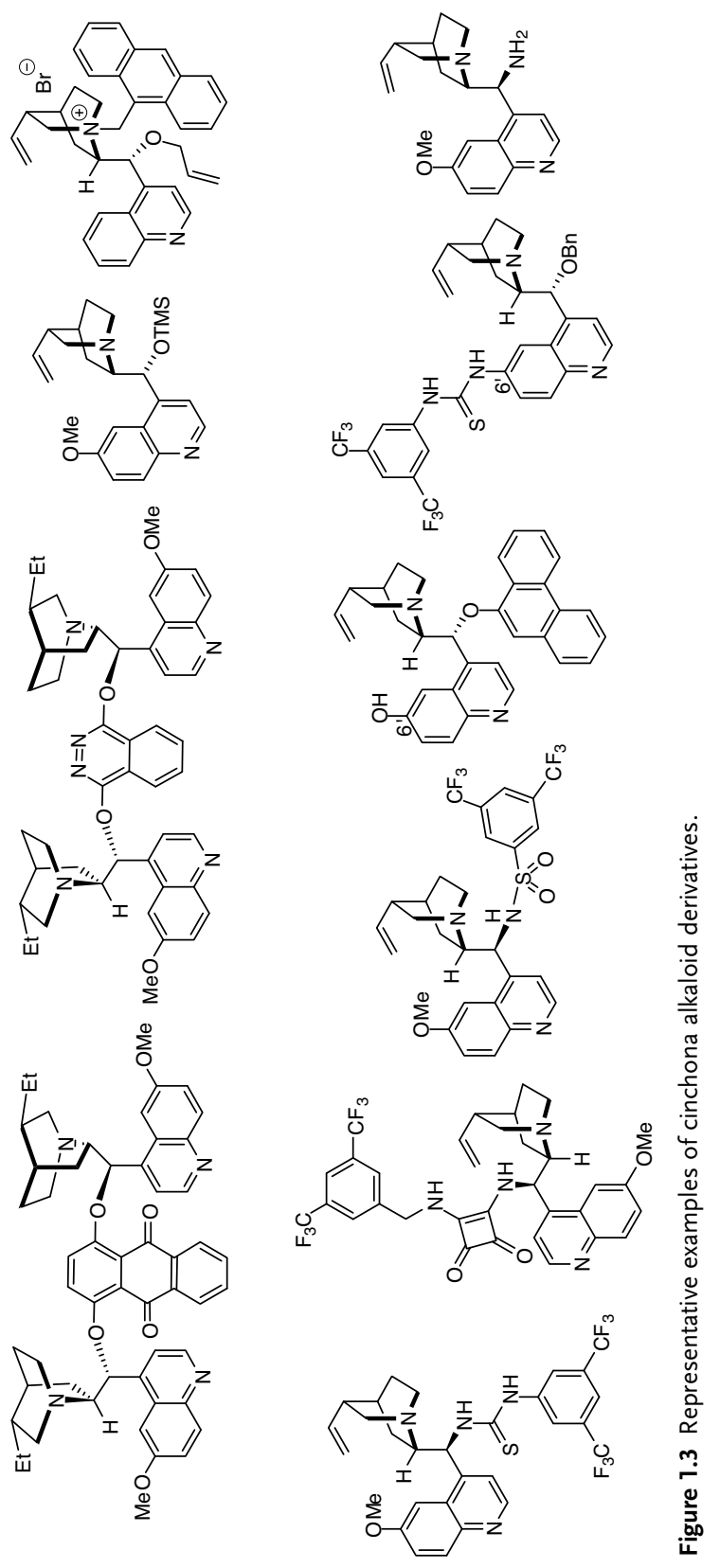
<smiles>C=CC1CN2CCC1C[C@H]2[C@H](O)c1ccnc2ccc(OC)cc12</smiles>

anti-closed

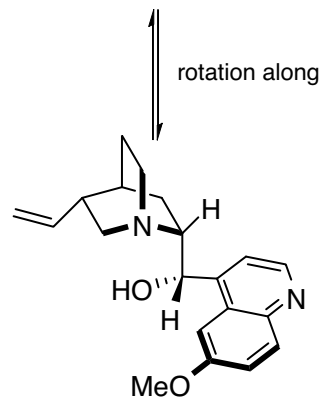

anti-open

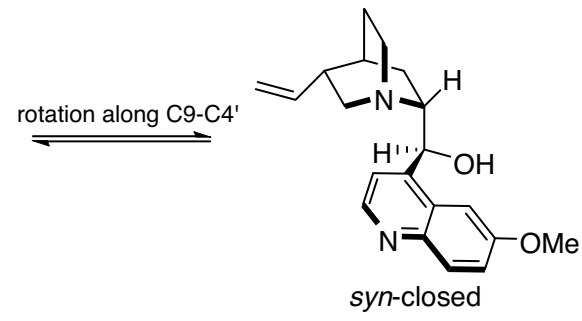

rotation along C8-C9

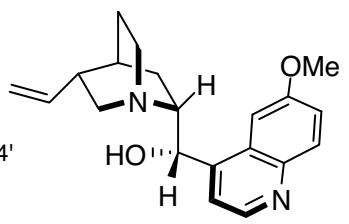

syn-open

Figure 1.4 The four conformers of quinidine showing the lowest energy.

room temperature. However, upon protonation, the anti-open conformation is observed exclusively [18]. The protonation of cinchona alkaloids appears to hinder their rotation around the $\mathrm{C} 4^{\prime}-\mathrm{C} 9$ and $\mathrm{C} 9-\mathrm{C} 8$ bonds and favor only a narrow range of the conformational space of the molecule [19].

The pivotal role of the conformational behavior of a cinchona alkaloid (e.g., cinchonidine) in its enantioselectivity was nicely illustrated in the platinum-catalyzed enantioselective hydrogenation of ketopantolactone in different solvents [18]. The achieved enantiomeric excess shows the same solvent dependence as the fraction of anti-open conformer in solution, suggesting that this conformer plays a crucial role in the enantiodifferentiation. As a more dramatic example, the solvent affects the absolute chirality of the product in the 1,3-hydron transfer reaction catalyzed by dihydroquinidine [20]. An NMR study revealed that the changes in the ratio between the two conformers of dihydroquinidine can explain the observed reversal of the sense of the enantioselectivity for this reaction when the solvent is changed from o-dichlorobenzene (open/closed 60:40) to DMSO (open/closed $\sim 20: 80$ ).

Undoubtedly, the modification of the structure of the cinchona alkaloid also has a significant effect on its conformational behavior in solution; esters [17] and 9-Ocarbamoyl derivatives [21] exist as a mixture of two major anti-closed and anti-open conformers, while C9 methyl ethers prefer an anti-closed arrangement in noncoordinating solvents [17]. Here again, protonation provides the anti-open conformation as the sole stable form [16b]. In addition to the solvent polarity, many other factors such as intermolecular interactions are also responsible for the complex conformational behavior of cinchona alkaloids in solution. 
Another characteristic structural feature of cinchona alkaloids is their multifunctional character and, thus, autoassociation phenomena are possible that could result in the strong dependency of their efficiency on the concentration and temperature [22, 23].

In 1969, Uskokovic and coworkers observed a concentration-dependent intermolecular interaction between dihydroquinine molecules [24]. The ${ }^{1} \mathrm{H}$ NMR spectra of (-)-dihydroquinine and racemic dihydroquinine are clearly different under concentrated conditions; that is, this molecule can generate its own nonequivalence. However, at high dilution $(0.01 \mathrm{M})$, this interaction disappeared. This indicates the existence of a self-association process mediated by intermolecular hydrogen bonding [24]. Moreover, the measurement of the molecular weight of quinine using osmometry conducted by Hiemstra and Wynberg revealed the presence of particles larger than monomeric quinine at $37^{\circ} \mathrm{C}$ for a $16 \mathrm{mM}$ solution in toluene. For concentrations less than $4 \mathrm{mM}$, on the other hand, quinine was almost completely monomeric [22].

In 1992, the coexistence of the monomer and dimers of quinine in chloroform solution was established by Salvadori and coworkers by investigating the temperature and concentration dependence of the NMR spectral parameters (chemical shift, NOE effect, relaxation time, etc.). The mole fraction of dimers was about $40 \%$ in $0.6 \mathrm{M}$ solution [25]. The structure of the dimer determined from NOESY and the relaxation rate data is given in Figure 1.5. As shown in Figure 1.5, the dimer of quinine was shown to be a $\pi-\pi$ complex with nearly parallel quinoline rings. A study carried out on the self-aggregation of chloroquine [26] gave similar results. Recently, the changes in the ${ }^{13} \mathrm{C}$ chemical shifts at various concentrations were also used to study the self-association of quinine. T-shaped dimers formed by the quinoline rings were proposed [27].

Quite recently, we also observed that quinine-based thiourea derivatives showed dramatic concentration and temperature effects on the enantioselectivity in the alcoholytic desymmetrization of meso-cyclic anhydrides, which can also be attributed to the self-association of the catalyst [23]. Of course, the possibility that the variation in

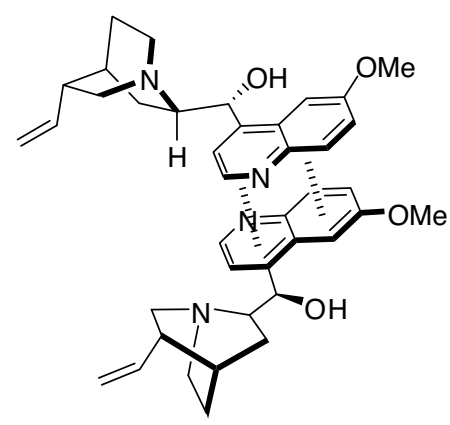

Figure 1.5 Conformation of quinine dimer from NMR results. 


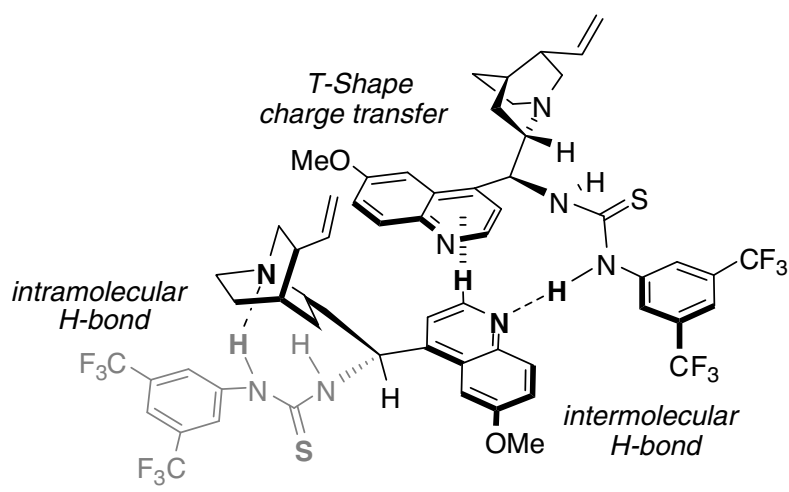

Figure 1.6 Dimeric structure of 9-epiquinine thiourea.

the enantioselectivity is caused by a concentration-dependent change in the conformational composition cannot be ruled out. However, according to the results obtained by Salvadori [25], at least in the case of the parent alkaloids, the conformation is similar in the dimer and monomer. Quite recently, Soós and coworkers proved by means of NMR NOESY experiments and computational studies that the quinine thiourea catalyst exists in a dimeric form under concentrated conditions due to H-bond and T-type $\pi-\pi$ interactions (Figure 1.6) [28].

As discussed, the solvent dipole moments, concentration, and temperature play a significant role in determining the structure of cinchona alkaloids and their derivatives in solution. In order to delineate the intimate details of the mechanism of action of cinchona alkaloids and their derivatives, a thorough understanding of their real structure in solution is needed. Furthermore, such detailed information on the real structure in solution would make it possible to develop new and more powerful chiral catalysts and discriminators.

\section{4}

\section{How This Book Is Organized}

The goal of this handbook is to provide up-to-date information on the whole spectrum of cinchona alkaloid chemistry. The authors have attempted to provide those who want to learn about the current state of the art on this topic and are willing to contribute actively to the extraordinary developments taking place in this field with an insider's view of this subject. This book is organized in four units, namely, the use of cinchona alkaloids as chirality inducers in metal-promoted reactions (Chapters 2-4), as chiral organocatalysts (Chapters 5-11), the organic chemistry of cinchona alkaloids themselves (Chapter 12), and their use as chiral discriminating agents in modern analysis (Chapter 13). In addition, a collection of carefully selected representative catalytic examples organized according to the reaction type is given in the form of an appendix. 


\section{References}

1 Garfield, S. (2000) Mauve, Faber \& Faber, London, p. 224.

2 From http://www.quinine-buchler.com/ cinchona.htm.

3 (a) Pasteur, L. (1853) Acad. Sci., 37, 162;

(b) Pasteur, L. (1853) Liebigs Ann. Chem., 88, 209.

4 (a) Newman, P. (1981) Optical Resolution Procedures for Chemical Compounds: Acids, vol. 2, Optical Resolution Information Center, Manhattan College, Riverdale, NY, pp. 7-22; (b) Jacques, J., Collet, A., and Wilen, S.H. (1981) Enantiomers, Racemates and Resolutions, John Wiley \& Sons, Inc., New York, pp. 254, 257; (c) Sheldon, R.A. (1993) Chirotechnology, Marcel Dekker, New York, Chapter 6; (d) Kozma, D. (2002) CRC Handbook of Optical Resolutions via Diastereomeric Salts, CRC Press, Boca Raton, FL.

5 Excellent reviews about quinine total synthesis with historical background: (a) Kaufmann, T.S. and Rúveda, E.A. (2005) Angew. Chem. Int. Ed., 44, 854; (b) Weinreb, S.M. (2001) Nature, 411, 429; (c) Nicolaou, K.C. and Sorensen, E.J. (1996) in Classics in Total Synthesis: Targets, Strategies, Methods, Wiley-VCH Verlag $\mathrm{GmbH}$, Weinheim, Chapter 15.

6 (a) Bredig, G. and Fiske, P.S. (1912) Biochem. Z., 46, 7; (b) Bredig, G. and Minaeff, M. (1932) Biochem. Z., 249, 241.

7 (a) Pracejus, H. (1960) Justus Liebigs Ann. Chem., 634, 9; (b) Pracejus, H. and Mätje, H. (1964) J. Prakt. Chem., 24, 195.

8 Wynberg, H. (1986) Top. Stereochem., 16, 87.

9 Selected reviews: (a) Kolb, H.C., VanNieuwenhze, M.S., and Sharpless, K.B. (1994) Chem. Rev., 94, 2483; (b) Kolb, H.C. and Sharpless, K.B. (1998) in Transition Metals for Organic Synthesis, vol. 2 (eds M. Beller and C. Bolm), Wiley-VCH Verlag GmbH, Weinheim, p. 219;(c) Johnson, R.A. and Sharpless, K.B. (2000) in Catalytic Asymmetric Synthesis, 2nd edn (ed. I. S Ojima), John
Wiley \& Sons, Inc., New York, p. 357;

(d) Beller, M. and Sharpless, K.B. (2002) in Applied Homogeneous Catalysis with Organometallic Compounds, 2nd edn, vol. 3 (eds B.H. Cornils and A. Wolfgang), Wiley-VCH Verlag GmbH, Weinheim, p. 1149.

10 (a) Berkessel, A. and Gröger, H. (2005) Asymmetric Organocatalysis, Wiley-VCH Verlag GmbH, Weinheim; (b) Dalko, P.I.(ed.) (2007) Enantioselective Organocatalysis, Wiley-VCH Verlag $\mathrm{GmbH}$, Weinheim; (c) List, B.(ed.) (2007) Chem. Rev., 107, 5413 (special issue on organocatalysis); (d) Houk, K.N. and List, B.(eds) (2004) Acc. Chem. Res., 37, 487 (special issue on asymmetric organocatalysis).

11 Berrisford, D.J., Bolm, C., and Sharpless, K.B. (1995) Angew. Chem. Int. Ed., 34, 1059.

12 For recent reviews on asymmetric phasetransfer catalysis, see (a) Maruoka, K.(ed.) (2008) Asymmetric Phase Transfer Catalysis, Wiley-VCH Verlag GmbH, Weinheim;

(b) Hashimoto, T. and Maruoka, K. (2007) Chem. Rev., 107, 5656.

13 For a recent review on organocatalysis via hydrogen bonding, see Doyle, A.G. and Jacobsen, E.N. (2007) Chem. Rev., 107, 5713.

14 For a recent review on enamine catalysis, see Mukherjee, S., Yang, J.W., Hoffmann, S., and List, B. (2007) Chem. Rev., 107, 5471.

15 For a recent review on iminium catalysis, see Erkkilä, A., Majander, I., and Pihko, P.M. (2007) Chem. Rev., 107, 5416.

16 (a) Dijkstra, G.D.H., Kellogg, R.M., and Wynberg, H. (1989) Recl. Trav. Chim. PaysBas, 108, 195; (b) Dijkstra, G.D.H., Kellogg, R.M., Wynberg, H., Svendsen, J.S., Marko, I., and Sharpless, K.B. (1989) J. Am. Chem. Soc., 111, 8069.

17 Dijkstra, G.D.H., Kellogg, R.M., and Wynberg, H. (1990) J. Org. Chem., 55, 6121.

18 Bürgi, T. and Baiker, A. (1998) J. Am. Chem. Soc., 120, 12920. 
19 Olsen, R.A., Borchardt, D., Mink, L., Agarwal, A., Mueller, L.J., and Zaera, F. (2006) J. Am. Chem. Soc., 128, 15594.

20 Aune, M., Gogoll, A., and Matsson, O. (1995) J. Org. Chem., 60, 1356.

21 Maier, N.M., Schefzick, S., Lombardo, G.M., Feliz, M., Rissanen, K., Lindner, W., and Lipkowitz, K.B. (2002) J. Am. Chem. Soc., 124, 8611.

22 Hiemstra, H. and Wynberg, H. (1981) J. Am. Chem. Soc., 103, 417.

23 Rho, H.S., Oh, S.H., Lee, J.W., Lee, J.Y., Chin, J., and Song, C.E. (2008) Chem. Commun., 1208.
24 Williams, T., Pitcher, R.G., Bommer, P., Gutzwiller, J., and Uskokovic, M. (1969) J. Am. Chem. Soc., 91, 1871

25 Uccello-Barretta, G., Bari, L.D., and Salvadori, P. (1992) Magn. Reson. Chem., 30, 1054.

26 Marchettini, N., Valensin, G., and Gaggelli, E. (1990) Biophys. Chem., 36, 65.

27 Casabianca, L.B. and de Dios, A.C. (2004) J. Phys. Chem. A, 108, 8505.

28 Tárkányi, G., Király, P., Varga, S., Vakulya, B., and Soós, T. (2008) Chem. Eur. J., 14, 6078. 